\title{
THE FIRST-2MASS RED QUASAR SURVEY. II. AN ANOMALOUSLY HIGH FRACTION OF LoBALs IN SEARCHES FOR DUST-REDDENED QUASARS*
}

\author{
Tanya Urrutia ${ }^{1,2,3}$, Robert H. Becker ${ }^{2,3}$, Richard L. White ${ }^{4}$, Eilat Glikman ${ }^{5}$, Mark Lacy ${ }^{1}$, Jacqueline Hodge $^{2}$, \\ AND Michael D. GREGG ${ }^{2,3}$ \\ ${ }^{1}$ Spitzer Science Center, MS 314-6, California Institute of Technology, 1200 E. California Boulevard, Pasadena, CA 91125, USA; mlacy@ipac.caltech.edu \\ 2 Department of Physics, University of California, One Shields Avenue, Davis, CA 95616, USA; hodge@ physics.ucdavis.edu \\ ${ }^{3}$ IGPP, L-413, Lawrence Livermore National Laboratory, Livermore, CA 94550, USA; bob@igpp.ucllnl.org, gregg@igpp.ucllnl.org \\ ${ }^{4}$ Space Telescope Science Institute, 3700 San Martin Drive, Baltimore, MD 21218, USA; rlw@ stsci.edu \\ 5 Astronomy Department, California Institute of Technology, Pasadena, CA 91125, USA; eilatg@astro.caltech.edu \\ Received 2008 February 22; accepted 2009 April 9; published 2009 May 27
}

\begin{abstract}
We present results on a survey to find extremely dust-reddened Type 1 quasars. Combining the FIRST radio survey, the 2MASS Infrared Survey and the Sloan Digital Sky Survey, we have selected a candidate list of 122 potential red quasars. With more than $80 \%$ spectroscopically identified objects, well over $50 \%$ are classified as dust-reddened Type 1 quasars, whose reddenings $(E(B-V))$ range from approximately 0.1 to 1.5 mag. They lie well off the color selection windows usually used to detect quasars and many fall within the stellar locus, which would have made it impossible to find these objects with traditional color selection techniques. The reddenings found are much more consistent with obscuration happening in the host galaxy rather than stemming from the dust torus. We find an unusually high fraction of broad absorption line (BAL) quasars at high redshift, all but one of them belonging to the low-ionization BAL (LoBAL) class and many also showing absorption in the metastable Fe II line (FeLoBAL). The discovery of further examples of dust-reddened LoBAL quasars provides more support for the hypothesis that BAL quasars (at least LoBAL quasars) represent an early stage in the lifetime of the quasar. The fact that we see such a high fraction of BALs could indicate that the quasar is in a young phase in which quasar feedback from the BAL winds is suppressing star formation in the host galaxy.
\end{abstract}

Key words: galaxies: active - galaxies: evolution - quasars: absorption lines - quasars: general

Online-only material: color figures, machine-readable table

\section{INTRODUCTION}

Since the identification of 3C 273 (Schmidt 1963), our understanding of quasars has evolved tremendously. There exists now a relatively robust quasar model in which different features of a quasar are explained invoking an orientation model (Antonucci 1993; Urry \& Padovani 1995). The orientation model describes the quasar by an accretion disk at the center and broad emission line clouds directly above and below the disk. The broad-line clouds can be shielded from our line of sight by a dusty donut-shaped torus coplanar with the disk. One explains whether or not an object is observed to have broad lines in the rest-frame optical wavelengths with the viewing angle of the active galactic nucleus (AGN). This model has also been successful in explaining the shape of the extragalactic Xray background spectrum, which peaks at $\sim 30 \mathrm{keV}$ (Treister \& Urry 2006; Gilli et al. 2007). There must be a large population of obscured quasars contributing to it to explain its spectrum peaking at such high energies.

The discovery of quasars through color selection techniques based on the typically blue spectrum of quasars has been extremely successful. It has resulted in a large output of optical spectra such as from the Sloan Digital Sky Survey (SDSS; Schneider et al. 2005), but also in quasar identifications in deep multiwavelength surveys (e.g., GOODS: Szokoly et al. 2004; Treister et al. 2004 and NOAODWFS: Hickox et al. 2007).

\footnotetext{
* Based on observations obtained with the W. M. Keck Observatory, which is jointly operated by the California Institute of Technology and the University of California.
}

By 2008 there are well over 100,000 quasars known, with another 100,000 likely candidates (Richards et al. 2004). However, the question of completeness of the current samples of quasars remains. Recent surveys have shown that optically selected quasars comprise less than half of the total quasar population (Martínez-Sansigre et al. 2005; Stern et al. 2007; Alonso-Herrero et al. 2006). Optical quasar surveys tend to miss dust-reddened quasars that have been found either with infrared (IR) surveys (Cutri et al. 2001; Lacy et al. 2004; Glikman et al. 2004, 2007), radio surveys (White et al. 2003), hard Xray surveys (Polletta et al. 2007) or surveys for high-ionization narrow-line objects (Zakamska et al. 2006). Furthermore, the distinction of unobscured/obscured has been proven to not be necessarily equivalent to Type 1 /Type 2 , as many dust-reddened and obscured quasars have been found with broad lines or featureless spectra (Alonso-Herrero et al. 2006; Martínez-Sansigre et al. 2006). Also, due to polarized scattering material in the polar directions the Type 1/Type 2 spectral features as an indicator of orientation may be ineffective; quasars with high dustcovering factors will always appear as Type 1 objects optically, regardless of orientation (Schmidt et al. 2007). In particular, broad absorption line quasar (BAL quasar) samples have redder colors than typical quasars (Sprayberry \& Foltz 1992; Reichard et al. 2003a) and higher X-ray column densities (Gallagher et al. 2006), also suggesting that a large fraction of this population is missing from optically selected samples.

This discrepancy in type of obscuration has been observationally recorded, especially in objects with different X-ray/mid-IR properties. In some AGNs which display Type 2 column densities in the X-rays, the mid-IR dust abundances are not correlated 
with those columns (Rigby et al. 2006). Furthermore, there are many high-luminosity objects in the mid-IR with AGN signatures, but with a prominent $\mathrm{Si}$ absorption feature at $10 \mu \mathrm{m}$, that do not exhibit strong absorption in the X-rays (Hao et al. 2007).

Current numerical simulations suggest that AGNs spend a large phase of their lifetime enshrouded by dust from the merging host galaxies (Hopkins et al. 2005; Li et al. 2007). This scenario has already been suggested in earlier studies, based on mid- and far-IR observational evidence (Sanders et al. 1988). AGNs in a growing phase are thought of as relatively luminous and heavily absorbed. Eventually the intense radiation field from the central engine destroys the surrounding obscuring material, and the AGN shines through unabsorbed (DiMatteo et al. 2005). These studies suggest that a non-negligible fraction of AGN are obscured by dust in the young merging host galaxy rather than a torus. In this picture, the quasar is also responsible for quenching some amount of the massive star formation occurring in the bulge of the host galaxy. Although there is a strong debate over how much influence this "quasar feedback" has on the host galaxy, these scenarios often invoke strong quasar winds that persist well outside the galaxy. BALs, the quasar phenomenon that we associate with very strong quasar winds, do not seem to have an evolutionary nature, but rather are explained by an "orientation hypothesis" (Weymann et al. 1991; Gallagher et al. 2007). However, the special cases of low-ionization BALs (LoBALs; showing broad absorption troughs in the Mg II line) and especially Fe II low-ionization BALs (FeLoBALs; with absorption troughs in the metastable Fe II line) are often invoked as classes of BALs that might be explained by a "youth hypothesis" in which BALs are at an early stage of their evolution in their quasar lifetime (Voit et al. 1993; Becker et al. 2000; Trump et al. 2006). Early studies of LoBALs show them to have larger dust covering fractions than standard BAL quasi-stellar objects (QSOs) and "normal" QSOs (Boroson \& Meyers 1992). Also for the FeLoBALs, radiative transfer spectral synthesis models show them to have large covering fractions for their outflows (Casebeer et al. 2008), and MIPS photometry shows them to be very luminous in the mid-IR, probably due to star formation (Farrah et al. 2007). Unfortunately, so far there is not conclusive evidence for polycyclic aromatic hydrocarbon (PAH) emission signifying star formation in BAL quasars, but a comprehensive unbiased sample, especially of LoBALs, is missing (Shi et al. 2006, 2007).

Finding dust-obscured quasars in the optical has proven to be a difficult task, as dust suppresses mostly ultraviolet (UV) and optical light. Since we want to study quasars at high redshift where the star formation rate and quasar fraction peak, the task is even more difficult, as the optical regime now covers mostly rest-frame UV light that is suppressed by several magnitudes from the dust. Nevertheless, Glikman et al. (2004, 2007; hereafter F2M) conducted a survey to find these dustreddened and -obscured quasars using the Two Micron All Sky Survey (2MASS) near-IR survey (Skrutskie et al. 1995) and the FIRST radio survey (Becker et al. 1995) and following up spectroscopically. This F2M survey largely concluded that ultraviolet excess (UVX) and optical color-selected sample in the optical miss $\geqslant 10 \%$ of the quasar population and that the red quasar population constitutes $>20 \%$ of the total quasar population.

In this paper, we describe a follow-up to the FIRST-2MASS survey described in F2M to find dust-reddened quasars using the FIRST radio survey (Becker et al. 1995) and the 2MASS Infrared Survey (Skrutskie et al. 1995) but with the additional photometry of the SDSS. It expands the original survey area, but only explores the objects with the reddest $r^{\prime}-K$ colors. Throughout this paper, we use a flat universe with $H_{0}=$ $70 \mathrm{~km}^{-1} \mathrm{Mpc}^{-1}, \Omega_{\Lambda}=0.7$ cosmology.

\section{OBSERVATIONS AND DATA ANALYSIS}

\subsection{Sample Definition}

To create a list of candidate red quasars, the 2MASS All-Sky Point Source Catalog (PSC) available at IPAC was matched to the FIRST source catalog within $2^{\prime \prime}$. In the initial F2M selection, the rate of correct matches drops significantly beyond $2^{\prime \prime}$, so we adopted this limit as well, reaching greater than $95 \%$ completeness at $2^{\prime \prime}$. If there were two or more matches within $2^{\prime \prime}$, then the closest match was taken. This initial matching ensures that we have bright IR sources with radio detections, which will remove most of the stars, since they usually do not have strong radio emission. We caution that radio selection may be introducing a bias, as quasars with higher radio luminosity have a tendency to show redder colors than quasars with very low radio luminosities (see Figure 13 of White et al. 2007).

The initial 2MASS catalog contains close to 471 million sources with near-IR magnitudes in the $J, H$, and $K_{s}$ bands. This survey is $99 \%$ complete to a magnitude limit at $10 \sigma$ of $J=15.8$, $H=15.1$, and $K=14.3$, although fainter sources are included in the catalog all the way down to $K \sim 16.0$. The FIRST catalog has more than 810,000 sources with $\sim 0$ '.5 positional accuracy. The 3 minute snapshot integration time yields a typical rms of $0.15 \mathrm{mJy}$, which gives significant detections for the catalog at the $5 \sigma$ level of $1 \mathrm{mJy}$. We obtained a list of 66,699 matches between these two catalogs within $2^{\prime \prime}$ and named the catalog FIRST-2MASS.

We then matched the FIRST-2MASS catalog to the approximately 215 million sources in the DR5 SDSS imaging catalog. We again matched them within $2^{\prime \prime}$ using the FIRST position as the comparing position, and if there was more than one match within $2^{\prime \prime}$, we chose the closest match. This matching yielded 59,974 objects; we call the new catalog F2M-SDSS.

We now filter the F2M-SDSS catalog for objects that are very red, i.e., $r^{\prime}-K>5$. While this selection is different from the F2M criterion $(R-K>4)$, the Sloan $r^{\prime}$ filter is slightly bluer than the Johnson $R$ filter used in the F2M color selection and the SDSS uses AB magnitudes rather than Vega. Our $r^{\prime}-K>5$ criterion corresponds to roughly a $R-K>4.5$ for a fairly flat $\left(g^{\prime}-r^{\prime}\right)$ color as described in Windhorst et al. (1991). Also, we opted to go for even redder objects since we are using the full 2MASS source catalog and our resultant FIRST-2MASS catalog is much larger. This extremely red color filter leaves us with 603 objects. Since the SDSS photometric limits in the $r^{\prime}$ band are around $23.1 \mathrm{mag}$, we should with good confidence have completeness in the optical, as a color of $r^{\prime}-K>8$ is very unlikely. The intrinsic luminosity of objects with such large amounts of dust to reach $r^{\prime}-K>8$ colors and still be detected in the optical would be very high (e.g., $\sim 20 L^{*}$ intrinsic luminosity for a $z=1$ red bulge galaxy) and the number density of such luminous objects is very small.

We inspected these 603 objects by eye. Most of them are bright galaxies, which had optical misclassifications or very close double objects in which the color filter picked up the faint object but the FIRST coordinate matches the bright object much better. The remaining 157 objects are very compact; only two have Petrosian radii (Petrosian 1976) larger than $4 "$. We 


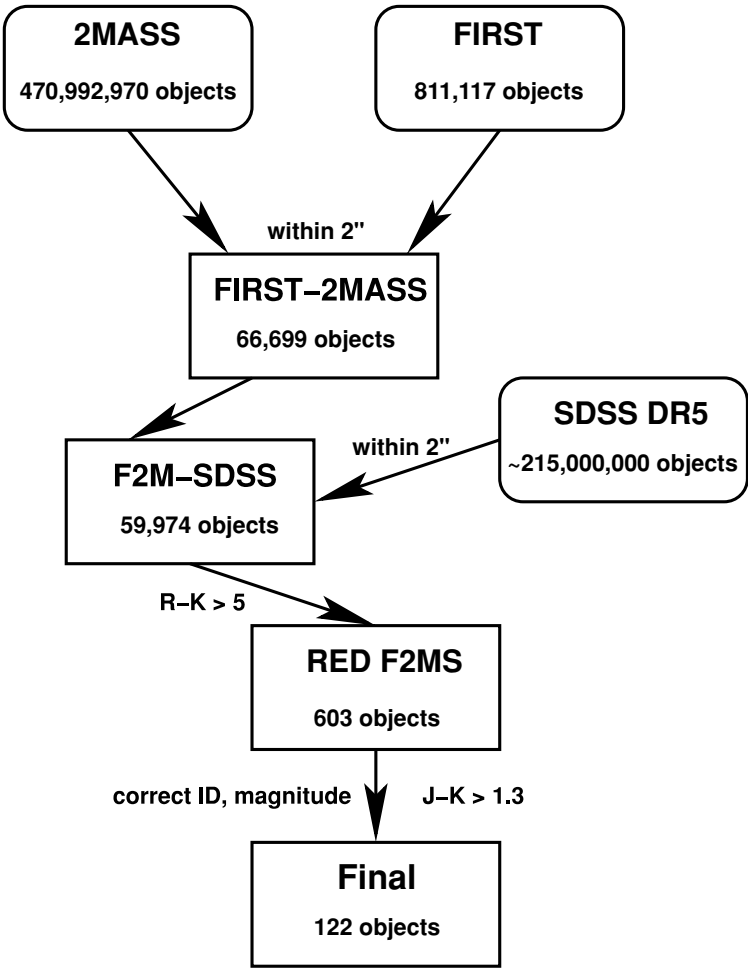

Figure 1. Selection process.

decided to build in an extra near-IR color filter $(J-K>1.3)$. We have spectra or identifications for 18 out of the 35 objects we discarded with this second color cut (13 M-stars, three galaxies, and two QSOs); this color regime is dominated by active radio stars. Quasars only contribute negligibly, so we do not have a large bias when discarding objects with this extra color cut. We are, however, missing some red quasars in this color range, such as the spectacular example of the radio-loud FeLoBAL FBQS1556+3517, which has a $J-K=1.12$ color (Becker et al. 1997). The $J-K>1.3$ color filter further reduces our catalog to 122 sources, which is our final F2MS catalog. The whole description is presented as a chart in Figure 1. The F2MS objects' coordinates and photometric properties are presented in Table 1.

In summary, the selection criteria are very similar to those determined in F2M, but we emphasize on only the reddest objects and complement with the five-band color information that SDSS provides. Because of the close overlap with the FIRST-2MASS survey, it is only natural that we recover many objects already cataloged in F2M. Overall, the catalogs have 33 $(27 \%)$ objects in common.

\subsection{Spectral Observations}

To identify the red quasar candidates, we conducted followup spectroscopy. Twenty of the 122 objects already had spectra taken with the SDSS fibers, so we did not duplicate those observations, even though in many cases the signal-to-noise ratio of the SDSS spectra was relatively low. Four objects had already been identified spectroscopically through the literature, so we decided against repeating those observations, even though we could not analyze the respective spectra. As this program has a large overlap with the F2M survey and both were done with some temporal overlap, many of the spectra presented here are the same as in the F2M survey. Our spectroscopic observations were conducted mostly at the $10 \mathrm{~m}$ Keck telescope with the ESI instrument in echellette mode with a $1^{\prime \prime}$ slit, giving a spectral resolution of $\sim 1.25 \AA$. Five objects were observed with the KAST spectrograph on the Shane $3 \mathrm{~m}$ telescope at the Lick observatory, at a spectral resolution of $\sim 2.35 \AA$. One object was observed with the Low Resolution Imaging Spectrometer (LRIS) instrument on the Keck $10 \mathrm{~m}$ telescope, one with the ALFOSC spectrograph on the $2.5 \mathrm{~m}$ Nordic Optical Telescope (NOT) and one with the Goodman Spectrograph on the $4.1 \mathrm{~m}$ SOAR telescope. Four of the objects have only near-IR spectra from the Spex instrument on the Infrared Telescope Facility 3 $\mathrm{m}$ telescope; we adopt their identification, but do not analyze their spectra. We now have spectra for 101 objects, making spectroscopic identification of the F2MS catalog over $80 \%$ complete.

While obtaining the redshift of an object is mostly straightforward, the true classification of an object is not trivial. This is especially true for spectra that show mixing of various classes of spectra, e.g., a poststarburst Balmer complex, but a very strong and broad $\mathrm{H} \beta$ line indicative of an AGN. Since most of our objects are at moderate redshift, we cannot use the ratios around $\mathrm{H} \alpha$ to classify the objects with the help of the BPT diagram. Following the conventions of F2M, we therefore make the following two cuts to help categorize the spectra. (1) If an object has an $\mathrm{O}_{\text {III }}{ }_{5007} / \mathrm{H} \beta$ flux ratio of $>2$, it is classified as an AGN. (2) If the $\mathrm{H} \beta$ line of an $\mathrm{AGN}$ is broader than $1000 \mathrm{~km} \mathrm{~s}^{-1}$, the object is classified as a broad-line quasar or Type 1 AGN. Note that we have two exceptions to the second rule (F2MS1618+3502 and F2MS1656+3821), as observations in the near-IR by F2M showed broad lines in their spectra and these were therefore classified as Type 1 quasars. For the highest redshift objects in which the $\mathrm{H} \beta$ line moves out of the optical spectrum, we use the $\mathrm{Mg}$ II line as a proxy for AGN activity. Also at high redshifts we have three spectra, which we would not have identified as quasars in the optical, but in which the near-IR spectra of F2M show broad emission lines and therefore are classified as red quasars (F2M0921+2707, F2M1313+3657, and F2M1344+2839). We also did not include known blazars such as F2MS1008+0621 as QSOs as they were probably included in the F2MS catalog due to their high variability rather than because they have an intrinsically red color. The identifications, redshifts, comments, and references to each source are presented in Table 1 and will be discussed further in Section 3.

\subsection{Reddening}

We measure the reddening of the objects classified as Type 1 quasars (QSO in Table 1) by fitting the red quasar spectra using the First Bright Quasar Survey (FBQS) composite spectrum (Brotherton et al. 2001) with a Small Magellanic Cloud (SMC) dust-reddening law. This FBQS spectrum was chosen because it represents an unreddened analog of our objects. For the reddening law, we use the relation from Fitzpatrick (1999):

$$
F_{\mathrm{o}}(\lambda)=F_{\mathrm{i}}(\lambda) 10^{-E(B-V) k(\lambda)} \times C
$$

with $F_{\mathrm{i}}(\lambda)$ being the intrinsic, unreddened (FBQS) and $F_{\mathrm{o}}(\lambda)$ the observed spectrum, and $C$ is a normalization constant. The SMC extinction curve $k(\lambda)$ lacks a significant $2175 \AA$ bump seen in the Galactic dust. We use the Galactic value of $R_{V}=$ 3.1 , therefore the extinction $A_{V}$ can be deduced by multiplying $E(B-V)$ by $R_{V}$. Variations in $R_{V}$ are only important above $7000 \AA$ rest frame, so if there are variations of the dust grain size, the deduced reddenings will only change for the lowest 
Table 1

Properties and Identification of Red Quasar Candidates

\begin{tabular}{|c|c|c|c|c|c|c|c|c|c|c|c|c|c|c|c|c|}
\hline No. & R.A. & Decl. & $F_{p k}$ & $u^{\prime}$ & $g^{\prime}$ & $r^{\prime}$ & $i^{\prime}$ & $J$ & $K$ & $r^{\prime}-K$ & $J-K$ & Spect. & Type & $z$ & Comments & Reference \\
\hline 001 & $00: 36: 59.85$ & $-01: 13: 32.30$ & 1.92 & 22.09 & 21.40 & 20.23 & 19.72 & 16.57 & 13.63 & 6.60 & 2.94 & ESI & QSO & 0.294 & Galaxy group in foreground, F2M & \\
\hline 002 & $01: 34: 35.68$ & $-09: 31: 03.00$ & 900.38 & 25.87 & 23.65 & 21.19 & 19.61 & 16.19 & 13.58 & 7.61 & 2.61 & NOT & QSO & 2.216 & Gravitational lens, F2M & Gr02,Wi02,+33 \\
\hline 003 & $01: 56: 47.61$ & $-00: 58: 07.46$ & 6.51 & 21.38 & 20.92 & 20.11 & 19.54 & 17.43 & 14.87 & 5.24 & 2.56 & ESI & NLAGN & 0.507 & & \\
\hline 004 & 07:30:51.03 & $+25: 38: 59.03$ & 1.23 & 22.01 & 20.59 & 19.49 & 19.07 & 16.85 & 14.15 & 5.34 & 2.70 & ESI & NLAGN & 0.289 & F2M & \\
\hline 005 & $07: 36: 24.18$ & $+42: 22: 17.15$ & 3.93 & 22.50 & 22.34 & 21.63 & 20.90 & 17.08 & 15.46 & 6.17 & 1.62 & None & $\cdots$ & $\ldots$ & & \\
\hline 006 & 07:38:05.95 & $+29: 51: 09.90$ & 2.64 & 20.89 & 22.12 & 20.82 & 19.15 & 16.94 & 15.28 & 5.54 & 1.66 & SDSS & Galaxy & 0.393 & F2M & \\
\hline 007 & $07: 38: 20.10$ & $+27: 50: 45.49$ & 2.64 & 23.78 & 22.73 & 20.74 & 19.48 & 17.05 & 15.28 & 5.46 & 1.77 & ESI & QSO & 1.985 & Mini-BAL, F2M & Gr02,Ri03,+4 \\
\hline 008 & 07:55:01.09 & $+40: 16: 32.16$ & 1.22 & 22.68 & 21.71 & 20.22 & 19.58 & 17.43 & 14.95 & 5.27 & 2.48 & SDSS & Galaxy & 0.381 & & \\
\hline 009 & $08: 01: 08.26$ & $+44: 01: 09.98$ & 211.67 & 21.03 & 20.37 & 19.84 & 19.44 & 16.45 & 14.83 & 5.01 & 1.62 & SDSS & BL Lac? & $1.072 ?$ & Featureless spectrum & \\
\hline 010 & 08:04:56.87 & $+19: 17: 26.85$ & 5.32 & 24.25 & 23.41 & 21.84 & 20.48 & 17.50 & 15.53 & 6.31 & 1.97 & None & $\cdots$ & $\cdots$ & & \\
\hline
\end{tabular}

Notes. Units of right ascension are hours, minutes, and seconds, and units of declination are degrees, arcminutes, and arcseconds. We use FIRST coordinates, which are in J2000 epoch.

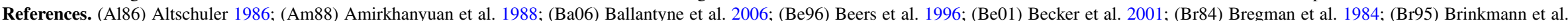

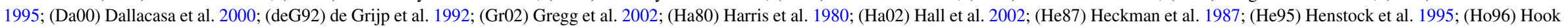

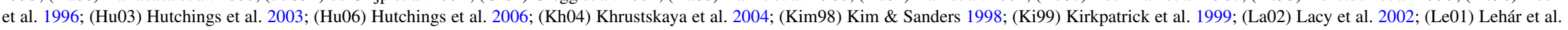

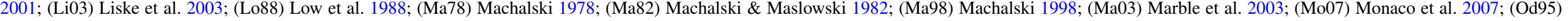

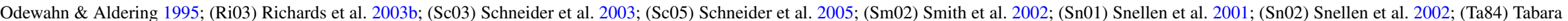
et al. 1984; (Ur05) Urrutia et al. 2005; (VV06) Véron-Cetty \& Véron 2006; (Wi83) Wills et al. 1983; (Wi98) Wilkinson et al. 1998; (Wi02) Winn et al. 2002; (Za03) Zakamska et al. 2003.

(This table is available in its entirety in a machine-readable form in the online journal. A portion is shown here for guidance regarding its form and content.) 
redshift objects. We chose to use the SMC extinction curve for all the quasars to deduce their reddenings, as that seems to model extinctions in QSOs the best (Richards et al. 2003b; White et al. 2003). There are some exceptions where the SMC extinction is not best (Czerny et al. 2004; Gaskell et al. 2004), but for the sake of comparison we apply the same dust law to all our Type 1 quasars. For a more detailed discussion on the dust law of red quasars and its possible variations, the reader can consult Section 5 of Paper I F2M. As shown in that discussion, the continuum reddening on average was lower than the Balmer reddenings, suggesting that the broad-line region is even more suppressed than the continuum. As the SDSS is designed as an extragalactic survey, Galactic extinction will not affect the reddening investigation much; Galactic extinction values were $<0.12 \mathrm{mag}$ in $r^{\prime}$ for all objects classified as quasars, which will have only a very small effect $(E(B-V)<0.04)$ on the reddening.

If an object has a reddening $E(B-V)>0.1$, we classify it as a red quasar. Only two Type 1 quasars are not classified as dust-reddened from our sample (F2MS1324+0537 and F2MS1102+5250), they are most likely highly variable objects whose flux varied between the 2MASS and SDSS observation epochs. The rest of the type 1 QSOs had dust reddenings significant enough to be classified as red in the range $E(B-V)=0.12-1.51$ (Table 2).

These reddenings are significant enough that the very red colors used in the selection process could not solely have come from a "red" spectral slope as is the case in some of the SDSS red quasars (Richards et al. 2003b). As discussed in Glikman et al. (2004, 2007), the reddening is also unlikely to come from a strong synchrotron component, so overall the red spectral slope is consistent with dust reddening. In addition, the reddenings can even be underestimated in some cases as the quasar is so obscured in the UV that there is significant host galaxy contribution to the spectrum (Urrutia et al. 2008). The spectra of the red quasars can be seen ordered by decreasing redshift in Figure 2.

\section{RESULTS}

The optical identification of the F2MS objects yielded the following classifications: 56 red quasars, among them $12 \mathrm{BAL}$ quasars, 11 narrow-line AGNs (NLAGN), 15 galaxies (either early type or starburst), 10 BL Lacs or blue unabsorbed quasars, and eight M-stars. Their redshift distribution is shown in Figure 3. The redshift distribution, especially of the quasars, peaks around $z \sim 0.7$. This is mostly a selection effect. At lower redshifts, a large number of low-luminosity object would not have been selected because the dominance of the host galaxy light prevents the quasar from making our color cuts. Host galaxies, even if red, do not show extreme $r^{\prime}-K>5$ colors so even if the quasar nucleus is very red the host galaxy will dilute the quasar's color contribution. At higher redshifts, the relatively shallow 2MASS magnitude limit means that only the most luminous objects are selected.

\subsection{Properties of the Red Quasars}

Once we have derived the reddening of the quasars, we can also obtain their intrinsic luminosities $\left(M_{i}\right)$ with the optical " $i$ " magnitude corrected for obscuration. They are presented in Table 2. We use the $K$-correction for quasars in the $i$ band provided by Richards et al. (2006), but use their adjustment to $z=0\left(M_{i}(z=0)=M_{i}(z=2)+0.596\right)$. The F2MS red
Table 2

Spectral Properties of the Quasars

\begin{tabular}{|c|c|c|c|c|}
\hline Source & $\begin{array}{c}\text { Redshift } \\
z\end{array}$ & $\begin{array}{c}E(B-V) \\
(\text { Rest })\end{array}$ & $\begin{array}{c}\mathrm{H} \beta \text { Width } \\
\left(\mathrm{km} \mathrm{s}^{-1}\right)\end{array}$ & $\begin{array}{c}\text { Absolute Mag. } \\
\qquad M_{i} \\
\end{array}$ \\
\hline F2MS0036-0113 & 0.294 & $0.96 \pm 0.10$ & $1150 \pm 100$ & -23.19 \\
\hline F2MS0134-0931 & 2.216 & $0.84 \pm 0.11$ & $\cdots$ & -31.44 \\
\hline F2MS0738+2750 & 1.985 & $0.67 \pm 0.11$ & $\cdots$ & -29.76 \\
\hline F2MS0825+4716 & 0.804 & $0.52 \pm 0.10$ & $1225 \pm 150$ & -27.65 \\
\hline F2MS0832+0509 & 1.070 & $0.14 \pm 0.04$ & $\cdots$ & -23.71 \\
\hline F2MS0834+3506 & 0.470 & $0.58 \pm 0.05$ & $2350 \pm 100$ & -24.25 \\
\hline F2MS0841+3604 & 0.552 & $1.34 \pm 0.11$ & $1500 \pm 500$ & -27.16 \\
\hline F2MS0854+3425 & 3.050 & $0.20 \pm 0.17$ & $\cdots$ & -27.23 \\
\hline F2MS0906+4952 & 1.635 & $0.12 \pm 0.07$ & $\ldots$ & -24.38 \\
\hline F2MS0911+0143 & 0.603 & $0.63 \pm 0.17$ & $1650 \pm 300$ & -24.20 \\
\hline F2MS0915+2418 & 0.842 & $0.36 \pm 0.12$ & $1900 \pm 200$ & -24.47 \\
\hline F2MS0921+1918 & 1.791 & $0.81 \pm 0.15$ & $\ldots$ & -29.13 \\
\hline F2MS0932+3854 & 0.506 & $0.85 \pm 0.12$ & $1000 \pm 150$ & -27.08 \\
\hline F2MS0943+5417 & 2.224 & $0.97 \pm 0.16$ & $\cdots$ & -31.07 \\
\hline F2MS1004+1229 & 2.658 & $0.76 \pm 0.24$ & $\cdots$ & -31.61 \\
\hline F2MS1005+6357 & 1.280 & $1.07 \pm 0.11$ & $\cdots$ & -28.31 \\
\hline F2MS1012+2825 & 0.937 & $0.82 \pm 0.10$ & $1500 \pm 200$ & -26.04 \\
\hline F2MS1030+5806 & 0.504 & $0.99 \pm 0.06$ & $4800 \pm 700$ & -26.55 \\
\hline F2MS1036+2828 & 1.760 & $0.61 \pm 0.07$ & $\cdots$ & -28.96 \\
\hline F2MS1113+1244 & 0.681 & $1.41 \pm 0.11$ & $3600 \pm 600$ & -28.37 \\
\hline F2MS1118-0033 & 0.686 & $0.85 \pm 0.11$ & $2100 \pm 500$ & -25.89 \\
\hline F2MS1121+2132 & 0.834 & $0.33 \pm 0.11$ & $1550 \pm 400$ & -23.88 \\
\hline F2MS1151+5359 & 0.780 & $0.42 \pm 0.08$ & $3300 \pm 500$ & -24.13 \\
\hline F2MS1243+1023 & 0.680 & $0.83 \pm 0.08$ & $1150 \pm 200$ & -25.77 \\
\hline F2MS1248+0531 & 0.740 & $0.26 \pm 0.07$ & $1250 \pm 200$ & -23.45 \\
\hline F2MS1256-0200 & 0.835 & $1.31 \pm 0.09$ & $3800 \pm 500$ & -27.92 \\
\hline F2MS1307+2338 & 0.275 & $0.75 \pm 0.09$ & $1200 \pm 200$ & -23.17 \\
\hline F2MS1309+6042 & 0.641 & $0.95 \pm 0.13$ & $2000 \pm 400$ & -24.87 \\
\hline F2MS1313+1453 & 0.584 & $1.12 \pm 0.15$ & $3100 \pm 500$ & -26.59 \\
\hline F2MS1341+3301 & 1.720 & $0.68 \pm 0.09$ & $\cdots$ & -28.78 \\
\hline F2MS1344+2839 & 1.770 & $0.55 \pm 0.12$ & $\cdots$ & -28.82 \\
\hline F2MS1353+3657 & 1.311 & $1.51 \pm 0.16$ & $\cdots$ & -29.81 \\
\hline F2MS1415+0903 & 0.654 & $0.45 \pm 0.11$ & $4300 \pm 500$ & -24.10 \\
\hline F2MS1434+0935 & 0.770 & $1.14 \pm 0.10$ & $2200 \pm 400$ & -27.20 \\
\hline F2MS1456+0114 & 2.378 & $0.45 \pm 0.16$ & $\cdots$ & -29.24 \\
\hline F2MS1500+0231 & 1.501 & $0.72 \pm 0.11$ & $\cdots$ & -28.44 \\
\hline F2MS1507+3129 & 0.988 & $0.74 \pm 0.09$ & $1700 \pm 250$ & -26.55 \\
\hline F2MS1523+0030 & 0.560 & $0.35 \pm 0.10$ & $1950 \pm 200$ & -23.24 \\
\hline F2MS1540+4923 & 0.696 & $0.97 \pm 0.10$ & $3500 \pm 300$ & -26.46 \\
\hline F2MS1548+0913 & 1.524 & $0.72 \pm 0.06$ & $\cdots$ & -28.36 \\
\hline F2MS1549+0306 & 0.679 & $0.64 \pm 0.08$ & $3600 \pm 500$ & -24.97 \\
\hline F2MS1551+0844 & 2.505 & $0.48 \pm 0.18$ & $\cdots$ & -29.76 \\
\hline F2MS1615+0318 & 0.424 & $0.42 \pm 0.06$ & $3400 \pm 300$ & -23.25 \\
\hline F2MS1618+3502 & 0.446 & $0.62 \pm 0.20$ & $700 *$ & -24.87 \\
\hline F2MS1638+2707 & 1.692 & $0.64 \pm 0.14$ & $\cdots$ & -27.76 \\
\hline F2MS1650+3242 & 1.059 & $0.79 \pm 0.06$ & $3800 \pm 500$ & -26.93 \\
\hline F2MS1656+3821 & 0.732 & $0.88 \pm 0.16$ & $350^{*}$ & -25.14 \\
\hline F2MS1708+2227 & 0.377 & $1.26 \pm 0.12$ & $3100 \pm 700$ & -24.69 \\
\hline F2MS1720+6156 & 0.727 & $1.50 \pm 0.09$ & $3900 \pm 400$ & -27.54 \\
\hline F2MS2152-0028 & 0.867 & $0.86 \pm 0.10$ & $2200 \pm 200$ & -25.77 \\
\hline F2MS2339-0912 & 0.660 & $1.25 \pm 0.06$ & $2500 \pm 300$ & -28.29 \\
\hline
\end{tabular}

quasars all lie above the quasar/Seyfert divide of $M_{i}=-22.5$ (Richards et al. 2006; Hao et al. 2005), we are dealing with a very luminous sample. Three quasars have absolute magnitudes $<-30$. These are known gravitational lenses whose computed luminosity is overestimated as a result of the lens magnification.

Figure 4 plots the absolute magnitude $M_{i}$ versus redshift. The most obscured objects $(E(B-V)>1.0)$ are shown as filled dots. Just as White et al. (2003) concludes, we can only detect the most obscured objects when they are so intrinsically luminous and/or when they lie at a relatively low redshift. We 

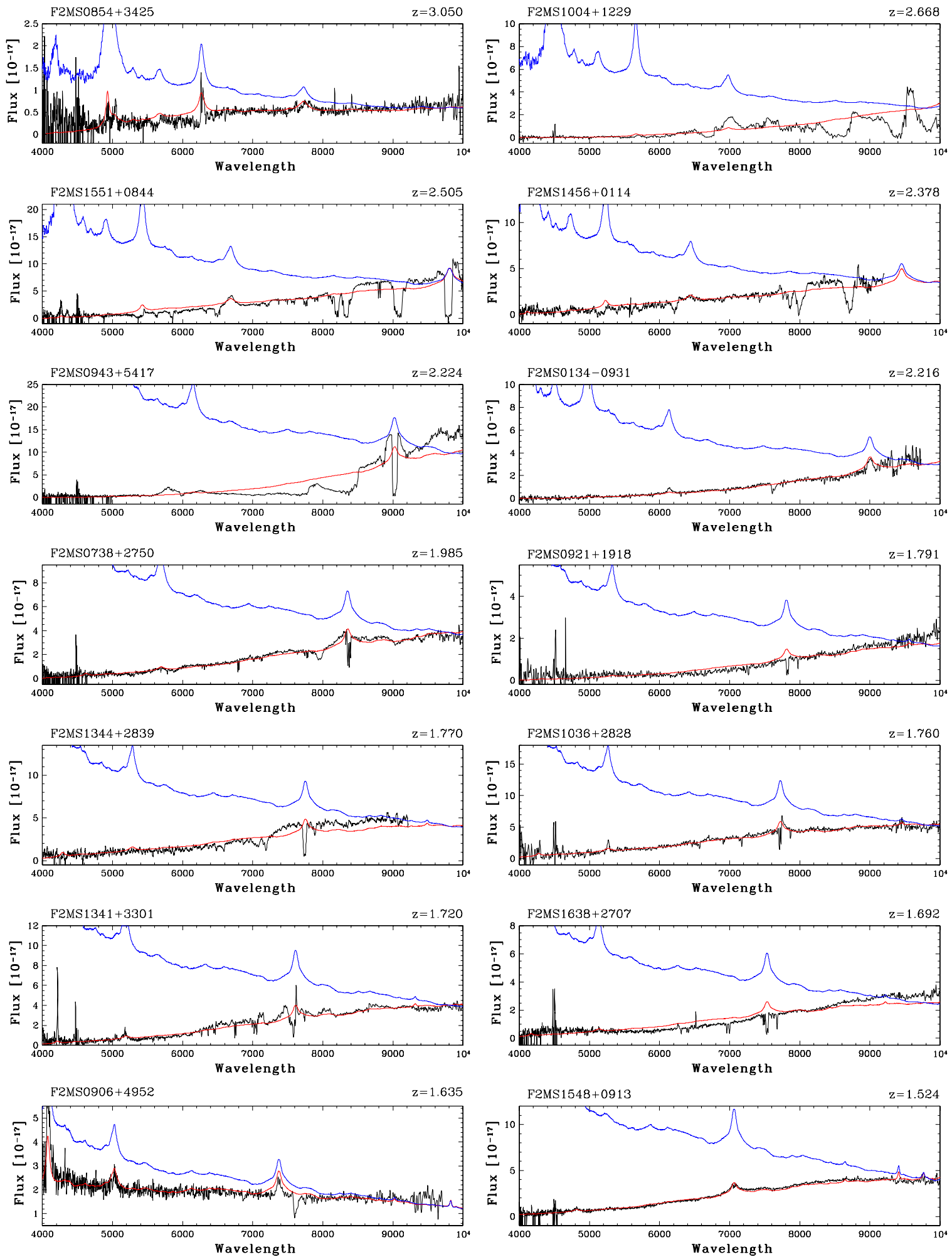

Figure 2. Redshift ordered spectra for the red type 1 QSOs in the F2MS sample. The wavelength range shown is from 4000 to $10000 \AA$ A. The black line is the observed quasar spectrum, the red line is the best-fit SMC reddening law applied to the FBQS composite. We show the FBQS spectrum as a blue line normalized to the reddened composite at $9800 \AA$ for presentation purposes only, normally the unreddened spectrum would have much higher flux.

(A color version of this figure is available in the online journal.)

are likely only detecting the most luminous tip of the red quasar iceberg. As expected, the highest redshift objects also have the highest absolute magnitudes. However, at low redshift there are not that many low-luminosity QSOs or obscured Seyferts. This can be a selection effect because the AGN component for Seyfert galaxies is so diluted by the host galaxy light, so that its colors are not quasar like (e.g., Satyapal et al. 2008). However, the steep rise in luminosity in Figure 4 also brings up the question of whether they have the same luminosity function as unobscured quasars. 

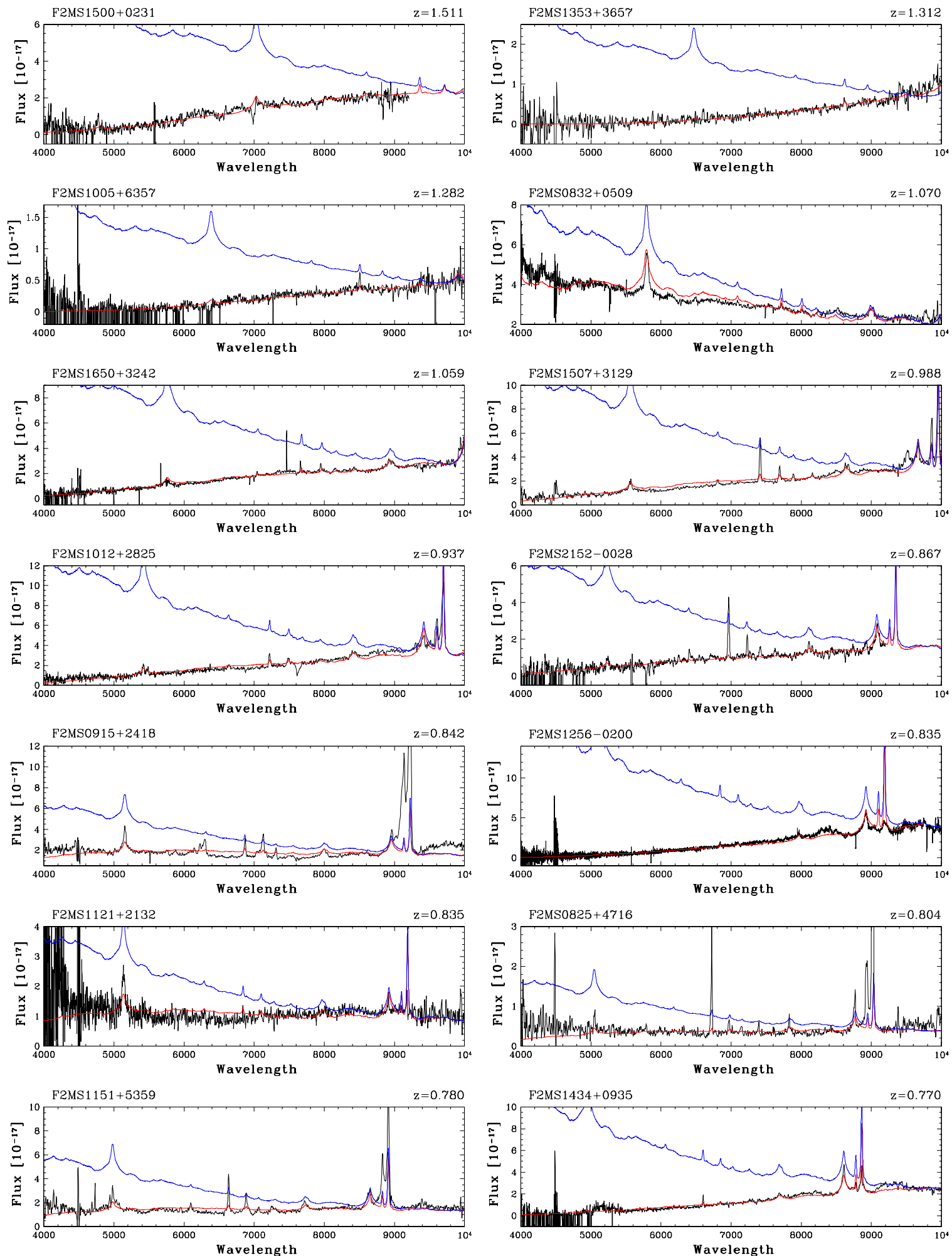

Figure 2. (Continued)

At first it would seem contradictory to claim that they do not, yet if we believe that dust-reddened quasars are in a young dust-enshrouded evolutionary phase, this phase would be highly intrinsically luminous as the central engine is accreting at high efficiencies (Hopkins et al. 2006). Some Spitzer-selected red QSOs also are only found at very high intrinsic luminosities (Polletta et al. 2008). Yet the results are not very conclusive, and there are counterexamples to this claim (Lacy et al. 2007).
Most of our sources have magnitudes that fall below the normal cutoff $(i=19.1)$ for spectroscopy with the SDSS. Some of them were targeted for spectroscopy as potential highredshift quasars or serendipitous radio source identifications. Of our 122 targets, $49(40 \%)$ had flags that would select them for potential QSO target follow-up. Therefore, optical surveys such as the SDSS would miss over $60 \%$ of these objects with traditional color selection algorithms. Richards et al. (2004) 

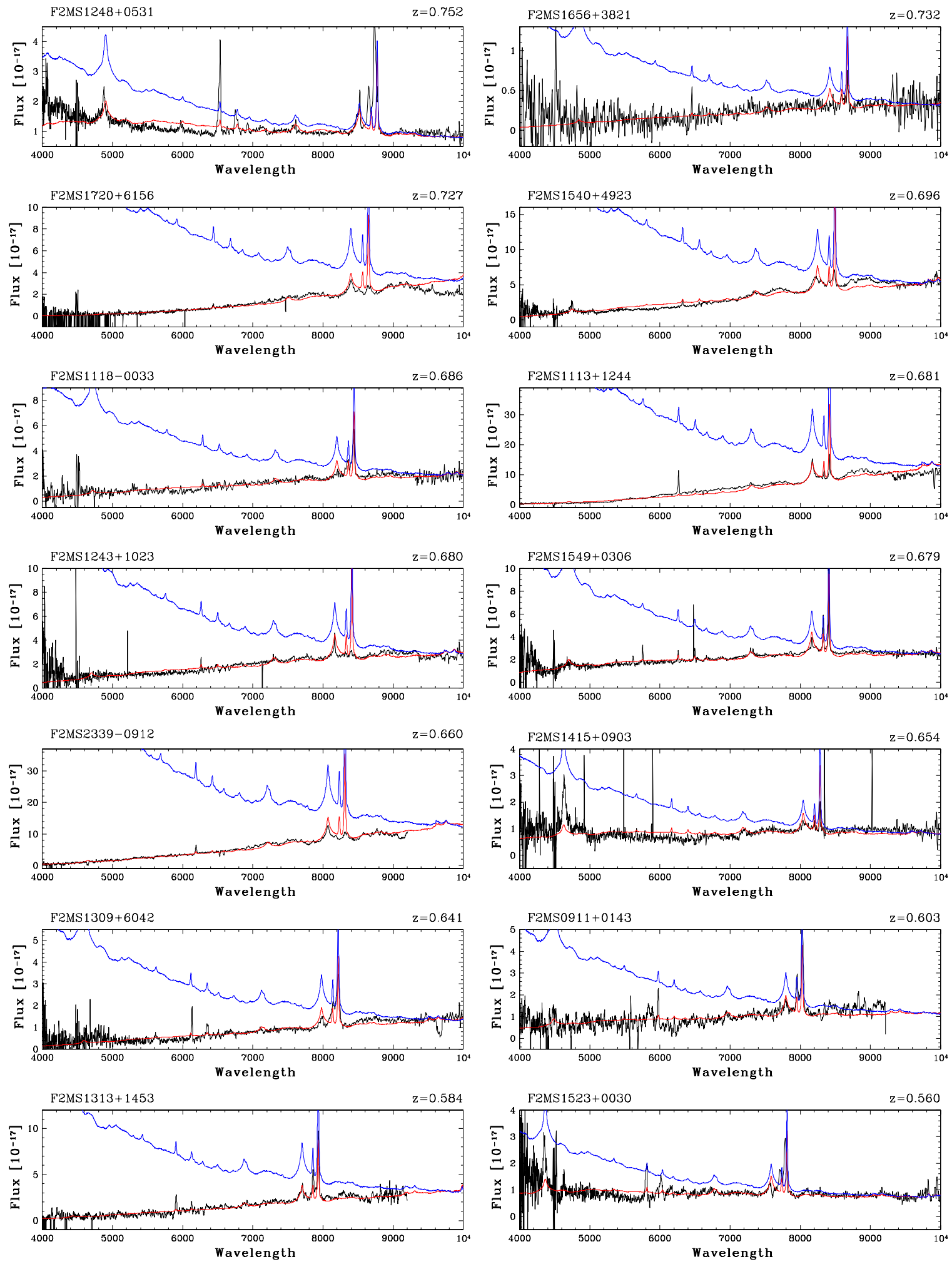

Figure 2. (Continued)

have undertaken a project to determine if even fainter parts of the color space are suitable for finding quasars, and they have potentially found over 100,000 QSOs in this manner. Figure 5 presents the SDSS colors of our red QSOs, plotted with red squares, the BALQSOs are denoted with a purple square. The small black dots denote the colors of the DR2 stars taken from the SDSS Web site. The blue box in the image is representative of the peak contour from Richards et al. (2004). Note that most of the F2MS red quasars and all of the F2MS BALQSOs lie outside that box. Furthermore, many of the F2MS red quasars lie on the stellar locus for different color-color plots, making optical selection algorithms unsuitable for identifying these objects. To include these objects, one needs near- and mid-IR photometry, which is insensitive to this amount of dust reddening (e.g., Lacy et al. 2004; Stern et al. 2007). These optical selection caveats are taken into account for deep fields such as the Spitzer First Look 

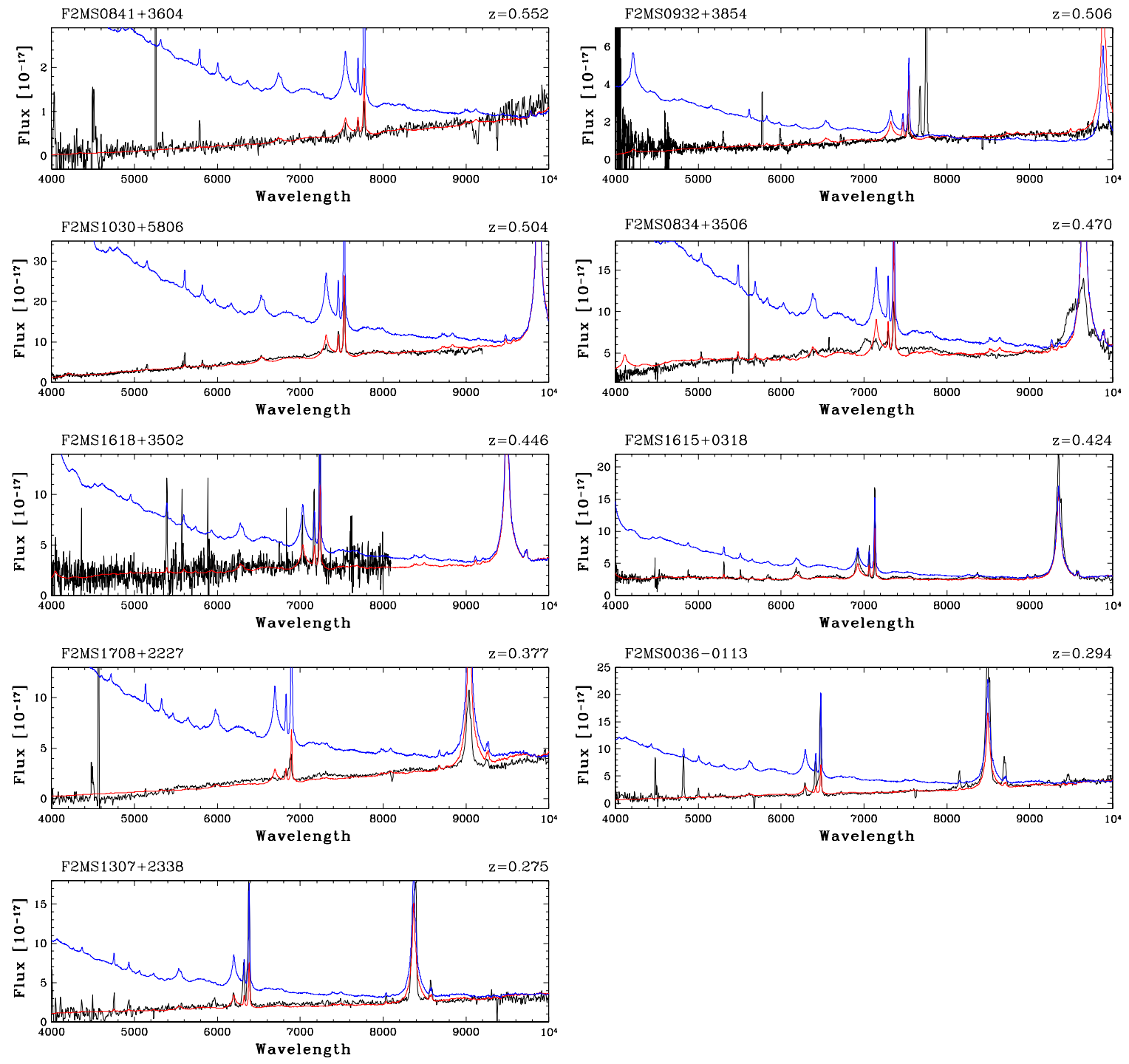

Figure 2. (Continued)

Survey, which has multiwavelength observations (Richards et al. 2009).

\subsection{An Anomalously High Fraction of Broad Absorption Line Quasars}

A BAL quasar is defined to be any quasar that shows significant broad absorption blueward of various quasar emission lines, such as $\mathrm{Mg}$ II $\lambda 2800$ or C IV $\lambda 1549$. BALs usually can only be identified at the high redshifts where the absorption troughs, usually present in the rest-frame UV part of the spectrum, are redshifted into the optical regime. For LoBALs, with their absorption troughs blueward of the $\mathrm{Mg}$ II line, this requires a redshift range of $0.9<z<2.6$ for the typical ESI spectral coverage of 4000-10000 A. While there are some lower redshift objects which show the Mg II emission line, ESI is not optimal in those wavelength regimes to determine absorption troughs; there is an Echelle break around $4500 \AA$, and below $5000 \AA$ the error spectrum rises steeply. LoBALs can also be identified by their Al III $\lambda 1859$ absorption at higher redshifts. For HiBALs, which only show prominent absorption troughs in the high-ionization lines such as $\mathrm{C}$ IV, we usually need redshifts of $z>1.7$; although even at these redshifts the flux in the blue part of the spectrum is suppressed as our objects have large dust reddenings. For the F2MS sample, 21 red quasars fall into the redshift range where BALs can be identified.

The definition of the Balnicity index was selected so that host and intervening narrow absorption systems are not selected erroneously. The traditional Balnicity index is defined by Weymann et al. (1991) as

$$
\mathrm{BI}=\int_{3000}^{25,000}\left[1-\frac{f(\mu)}{0.9}\right] C d \mu,
$$

where $f(\mu)$ is the normalized flux, $C=1$ at absorption through velocities more than $2000 \mathrm{~km} \mathrm{~s}^{-1}$ from the start of a continuous trough and zero elsewhere. Usually, uncertainties in the continuum dominate over the formal error, so we do not include errors for our estimated Balnicities.

With somewhat better resolution data and better continuum fitting techniques, Trump et al. (2006) decided to use the absorption index (AI) to identify BALQSOs. The AI is defined by Hall et al. (2002) as

$$
\mathrm{AI}=\int_{0}^{29,000}[1-f(\mu)] C^{\prime} d \mu,
$$




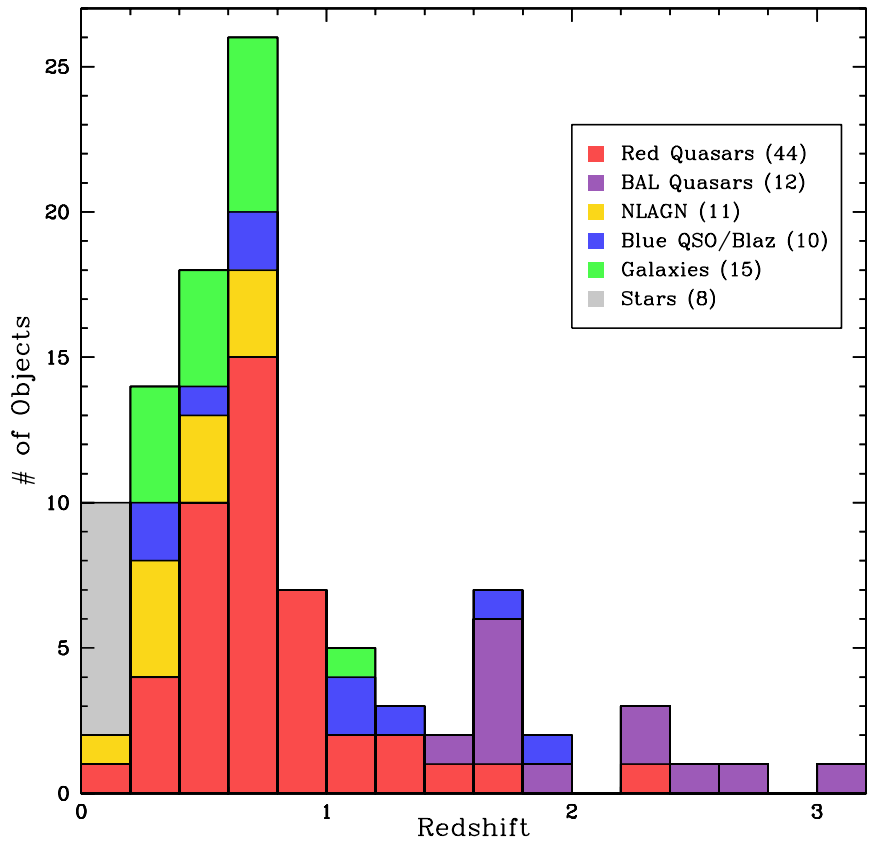

Figure 3. Redshift distribution of the spectroscopically identified objects. Note the high fraction of BAL quasars at high redshift.

(A color version of this figure is available in the online journal.)

where $f(\mu)$ is the normalized flux. $C^{\prime}=0$ except in continuous troughs with the minimum width of $1000 \mathrm{~km} \mathrm{~s}^{-1}$ and a minimum $10 \%$ depth, in which case $C^{\prime}=-1$.

There is still disagreement over the exact definition of a BAL. While the traditional, more conservative Balnicity index measure ensures that the absorption is from a nuclear outflow, and effectively excludes "associated absorbers," this definition could potentially exclude unusual or interesting BALs (e.g., Becker et al. 2000). The AI, on the other hand, allows for somewhat narrower troughs and absorption at the emission redshift. Knigge et al. (2008) warn that a BAL classification via the AI needs to be reconsidered, since its distribution is bimodal (Knigge et al. 2008). Trump et al. (2006) find that $26 \%$ of all QSOs are BALs if defined by the AI. This is higher than the $\sim 12 \%$ fraction determined using the traditional Balnicity index.

We fitted the continuum for the 21 red F2MS quasars that were in the redshift range suited to be identified as BALs. Our small sample size allowed us to adjust the continuum at the emission lines by hand rather than let an automated process run over it. We then normalized the spectra and calculated the Balnicity and AIs according to the formulae above. We used mostly the $\mathrm{Mg}$ II line as a proxy for Balnicity, since the $\mathrm{C}$ IV line was very noisy for the objects with $z<2$.

As mentioned before, the ESI spectrograph has a poor throughput at the blue end of the spectrum. Not only are the quasars very extinguished in that part, but the poor throughput in the near-UV from ESI makes the spectrum very noisy. It is very difficult to distinguish absorption $\mathrm{Mg}$ II troughs qualitatively below a redshift of 0.9 .

We also introduce the reduced chi-squared of the trough as defined by Trump et al. (2006):

$$
\chi_{\text {trough }}^{2}=\sum \frac{1}{N}\left[\frac{1-f(v)}{\sigma}\right]^{2},
$$

where $N$ is the number of pixels in the trough and $f(v)$ is the normalized template-subtracted flux. The higher the $\chi_{\text {trough }}^{2}$,

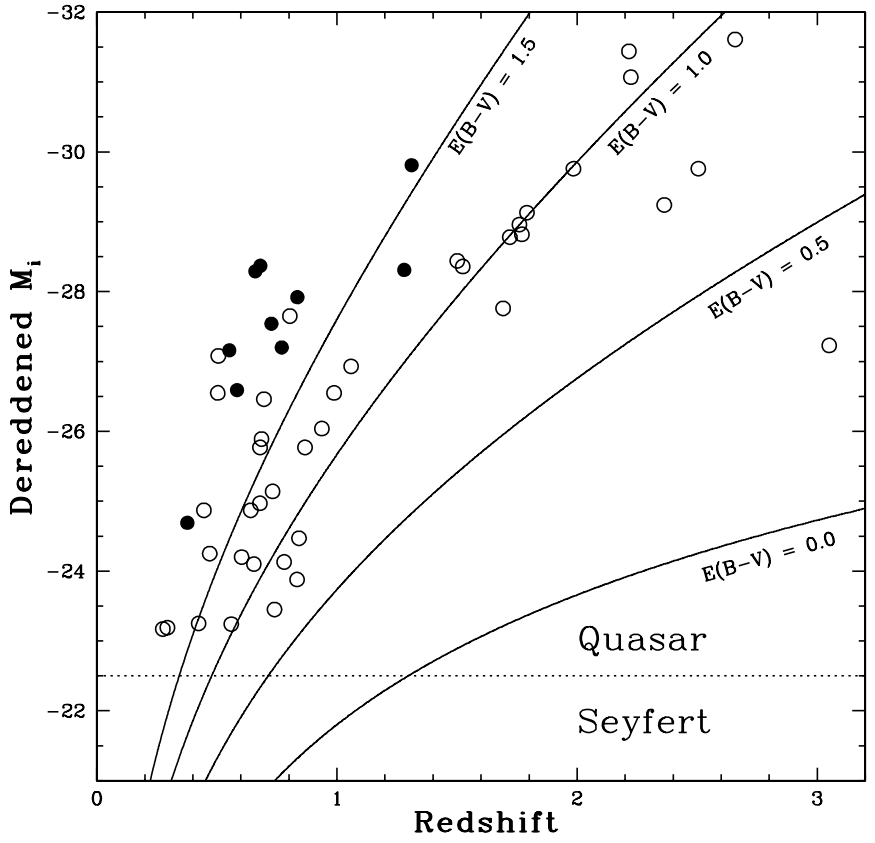

Figure 4. Redness-corrected absolute magnitude $\left(M_{i}\right)$ of the F2MS red quasars vs. redshift. The quasars with the highest reddenings $(E(B-V)>1.0)$ are indicated as filled dots. The curves show SDSS $i<22.3$ detection limit as a function of reddening $E(B-V)$. Note that at $z>2.0$ quasars with such large reddenings become undetectable, which tells us that we must be missing a large fraction of objects at lower luminosities and higher redshifts (also see White et al. 2003).

the less likely the trough is due to noise or a really shallow artificial trough introduced by a bad continuum fit. We require a $\chi_{\text {through }}^{2}>10$ to be considered true BAL troughs. We make one exception for F2MS1456+0114, whose C IV line trough has a $\chi_{\text {trough }}^{2}<10$, but shows such prominent Fe II absorption that it is also very likely to have very high $\mathrm{Mg}$ II absorption if it had spectral coverage there. For the calculation of the $\mathrm{Mg}$ II fraction, we do not include F2MS1353+3657 and F2MS1005+6357, which clearly have noise-dominated spectra, where the error is sometimes double than that of the signal. We do however include F2MS0906+4952, F2MS1507+3129, and F2MS1012+2825 as non-BALs; from visual inspection, we can deduce that the troughs are not real. However, we do note that there is generally a lack of BALs in our sample below redshift $z=1.5$. We do not know if that is a selection effect or if there are two different populations at high and low redshift.

Twelve of the $19(63 \%)$ eligible objects were identified as BALQSOs using the AI. However, five of the objects would have been rejected as a BAL by Weymann et al. (1991) since the absorption troughs were either too narrow (below $2000 \mathrm{~km} \mathrm{~s}^{-1}$, but above $1000 \mathrm{~km} \mathrm{~s}^{-1}$ ), or because they were so close to the emission redshift and could have been mistaken with absorbers associated with the host galaxy (although most host galaxies do not have such large velocity dispersions). We label these five objects as Mini-BALs, yet include them in the discussion about BALs. If we were to exclude the objects with zero BIs, seven out of 19 F2MS quasars within the appropriate redshift are BALs $(37 \%)$. Figure 6 shows the spectra of the BALs with the absorption troughs bolded in red. Non-BALs for a redshift $>0.9$ are shown in Figure 7, with the noisy spectra included for completeness.

Our conservative BAL fraction of $37 \%$ alone is already much higher than almost all other quasar surveys. But even more 

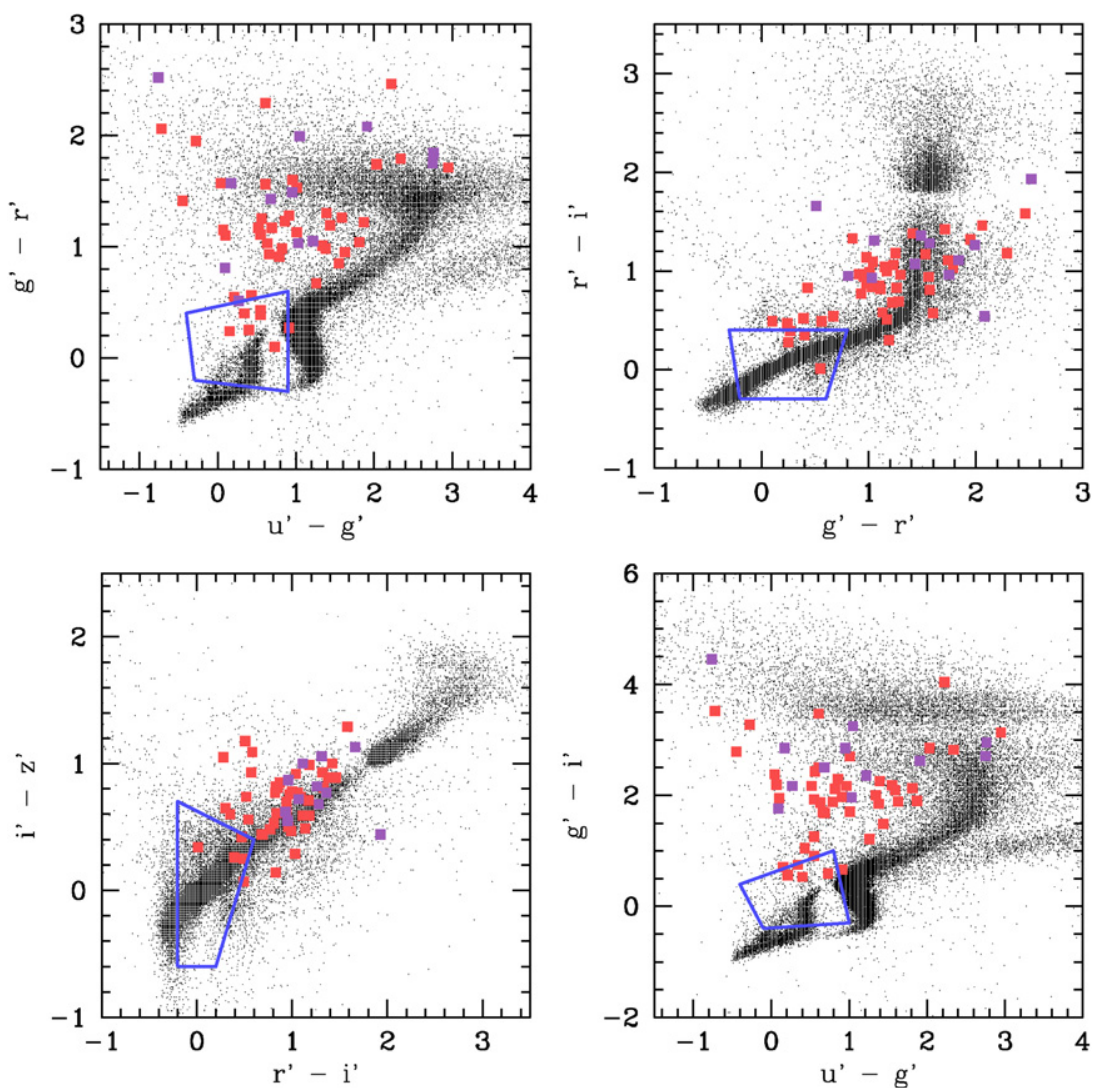

Figure 5. Various optical colors of the objects classified as red quasars and BAL quasars. The small black dots are the colors of stars in SDSS DR2. The blue boxes are the representation of the quasars contours of the SDSS $z<3$ from Richards et al. (2004). Note that most of our red quasars and all of the BAL quasars lie outside those boxes and that the red quasars often fall into the stellar locus making it difficult for typical quasar search algorithms to find these objects.

(A color version of this figure is available in the online journal.)

Table 3

Absorption Trough Properties of Quasars with $z>0.9$

\begin{tabular}{|c|c|c|c|c|c|}
\hline Source & Redshift & Balnicity & Absorption Index & $\chi_{\text {through }}^{2}$ & Type \\
\hline F2MS0854+3425 & 3.050 & $535^{\mathrm{a}}$ & $2077^{\mathrm{a}}$ & 11.65 & BAL/HiBAL (no Mg II) \\
\hline F2MS1004+1229 & 2.668 & 11540 & 17374 & 3062 & BAL/FeLoBAL \\
\hline F2MS1551+0844 & 2.505 & 941 & 6575 & 28.90 & BAL/FeLoBAL \\
\hline F2MS1456+0114 & 2.378 & $3991^{\mathrm{a}}$ & $6422^{\mathrm{a}}$ & 4.491 & BAL/FeLoBAL (based on Fe II) \\
\hline F2MS0943+5417 & 2.224 & 2539 & 8467 & 2231 & BAL/FeLoBAL \\
\hline F2MS0134-0931 & 2.216 & 0 & 0 & $\ldots$ & Non-BAL \\
\hline F2MS0738+2750 & 1.985 & 0 & 778 & 83.78 & Mini-Bal/LoBAL \\
\hline F2MS0921+1918 & 1.791 & 0 & 317 & 49.59 & Mini-Bal/FeLoBAL \\
\hline F2MS1344+2839 & 1.770 & 0 & 2059 & 12.39 & Mini-BAL/FeLoBAL \\
\hline F2MS1036+2828 & 1.761 & 0 & 363 & 1241 & Mini-BAL/FeLoBAL \\
\hline F2MS1341+3301 & 1.720 & 1725 & 6368 & 1031 & BAL/FeLoBAL \\
\hline F2MS1638+2707 & 1.692 & 0 & 579 & 217.1 & Mini-BAL/FeLoBAL \\
\hline F2MS0906+4952 & 1.635 & 0 & 77 & 1.421 & Non-BAL \\
\hline F2MS1548+0913 & 1.524 & 0 & 0 & $\ldots$ & Non-BAL \\
\hline $\mathrm{F} 2 \mathrm{MS} 1500+0231$ & 1.511 & 355 & 4232 & 10.61 & BAL/FeLoBAL \\
\hline F2MS1353+3657 & 1.312 & 4503 & 3111 & 1.166 & Too noisy \\
\hline F2MS1005+6357 & 1.282 & 6351 & 7309 & 4.512 & Too noisy \\
\hline F2MS0832+0509 & 1.070 & 0 & 0 & $\ldots$ & Non-BAL \\
\hline F2MS1650+3241 & 1.059 & 0 & 0 & $\ldots$ & Non-BAL \\
\hline F2MS1507+3129 & 0.988 & 0 & 616 & 6.933 & Non-BAL \\
\hline F2MS1012+2825 & 0.937 & 428 & 452 & 0.611 & Non-BAL \\
\hline
\end{tabular}

Note. ${ }^{a} \mathrm{C}$ IV derived Balnicities and AI, all other are for the Mg II line, which in most cases had much higher signal-to-noise ratio in the spectra.

interesting is the fact that all seven BALQSOs that had Mg II wavelength coverage were LoBALs. F2MS0854+3425 has no Mg II coverage, but shows no Al III $\lambda 1859$ absorption through, so it is most likely a HiBAL. Also remarkable is that all of the objects classified as LoBALs also were of the rare population of FeLoBALs. Our conservative LoBAL, and also FeLoBAL, fraction is $32 \%(6 / 19)$. Trump et al. (2006) find LoBALs and FeLoBALs fraction to be $0.5 \%$ and $0.3 \%$, respectively. This 2 
CIV Absorption troughs
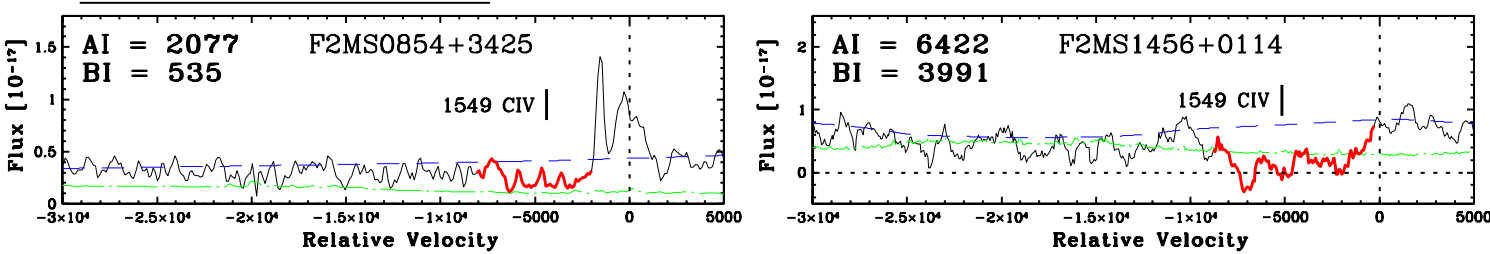

MgII Absorption troughs
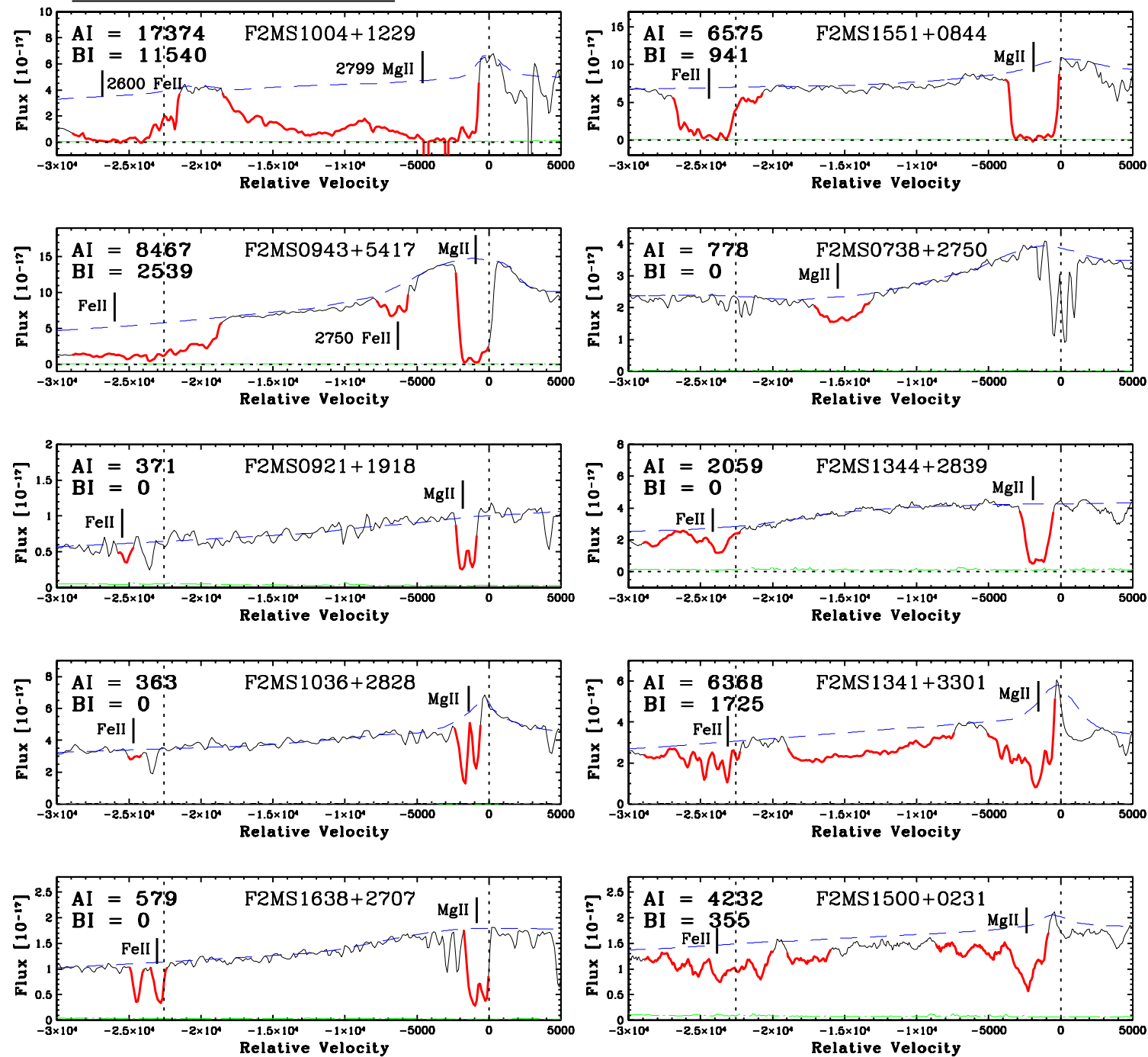

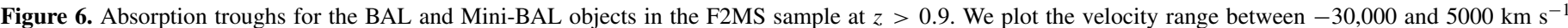

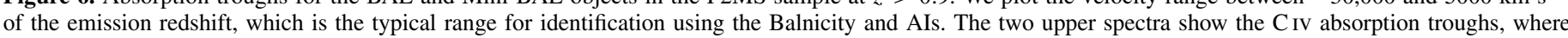

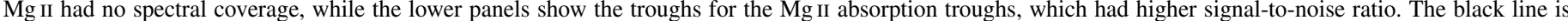

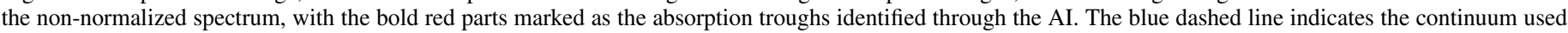

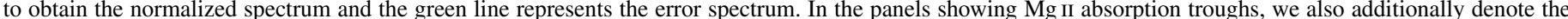

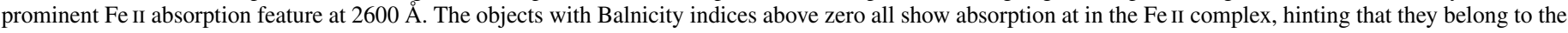
rare class of FeLoBALs.

(A color version of this figure is available in the online journal.)

order of magnitude discrepancy for $(\mathrm{Fe})$ LoBAL fraction is truly remarkable; dust-absorbed and -reddened quasars show a much higher than usual fraction of LoBALs than quasars selected in optical surveys.

\section{DISCUSSION}

We have expanded the original FIRST-2MASS red quasar survey to include the whole extent of the 2MASS PSC but only include objects which have a very red color $\left(r^{\prime}-K>5\right)$. This yielded 122 red quasar candidates. Spectroscopic follow-up on 100 of the sources revealed that well over $50 \%$ are red type 1 QSOs, consistent with the original FIRST-2MASS red quasar survey.

As described in Section 2.2, the deduced reddening is most likely due to dust absorption. The question remains where the obscuring dust is located. It could be in regions of the torus, where sublimation does not affect it, or it could be in a cold 
Non-BAL quasars
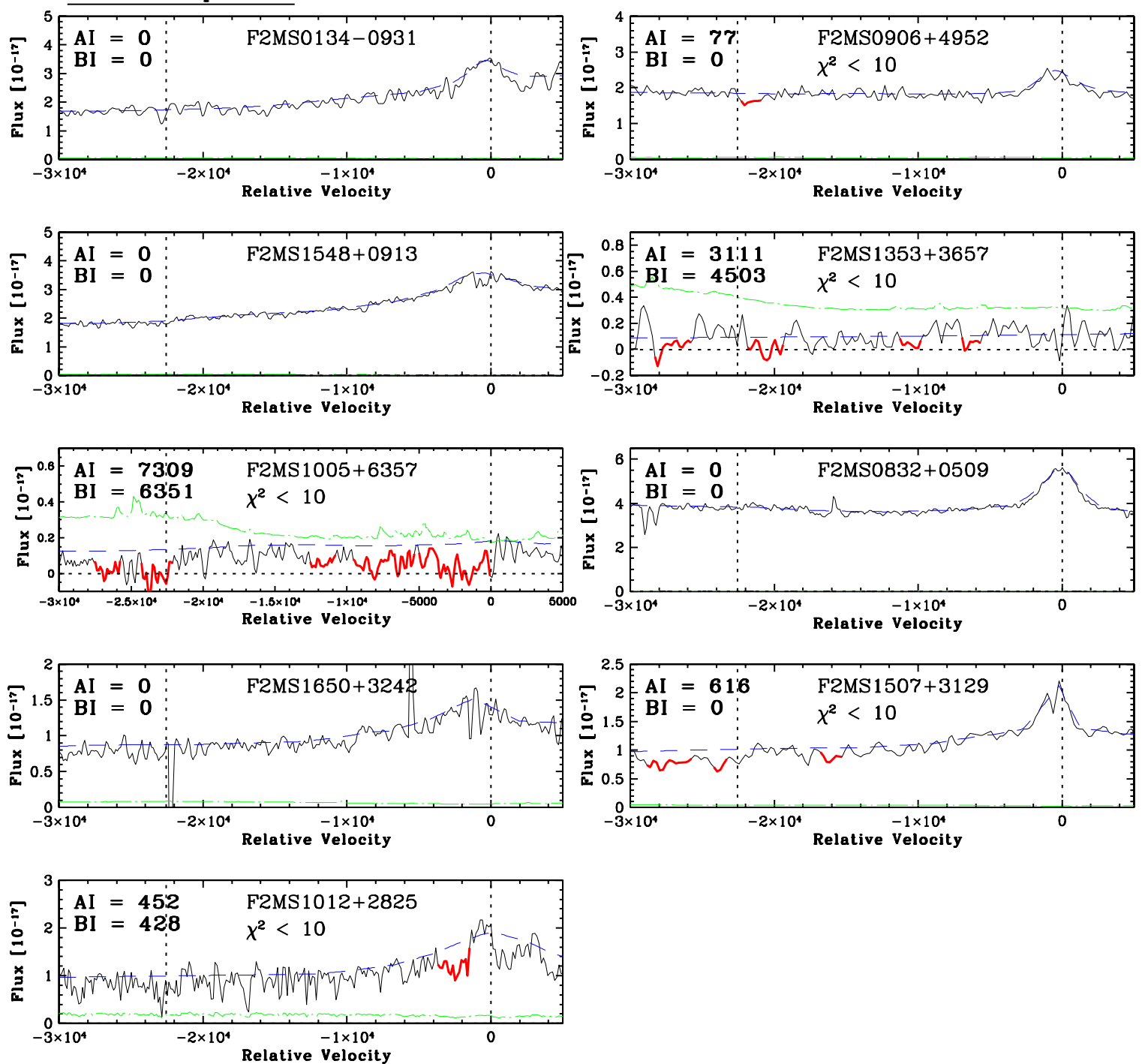

Figure 7. Same range and properties as Figure 6, only for the non-BAL quasars at $z>0.9$. The two dotted lines represent where blueward, the Mg II and Fe II absorption features, would lie. F2MS1353+3657 and F2MS1005+0231 are clearly dominated by noise, so we do not include them in our calculation for the BAL fraction.

(A color version of this figure is available in the online journal.)

outside absorber, i.e., the host galaxy. Some clues as to the nature of the extinction in these objects came from our Hubble Space Telescope (HST)/Advanced Camera for Surveys (ACS) study of a sample of 13 F2M quasars (those in the F2MS sample are labeled in Table 1). The images showed impressive evidence for a recent merger in an unusually high percentage $(85 \%)$ of the red quasars. Also, there was a weak correlation between the reddening and the amount of interaction displayed in the host galaxy measured by the Gini coefficient. These dust-reddened quasars may be young, recently ignited objects, where quasar feedback has yet to quench the star formation activity (Urrutia et al. 2008). Ongoing Spitzer IRS and MIPS observations of the HST/ACS sample should help to test this hypothesis, as we will be able to measure PAH strengths and $70 \mu \mathrm{m}$ dust emission likely associated with young star forming host galaxies. The Spitzer IRS spectra will also describe the absorbing dust properties better by analyzing the $9.7 \mu \mathrm{m}$ silicate absorption feature and relating that to our deduced reddening.
There is still debate over how biased our surveys are. Radioselected samples tend to have redder colors. However, IRselected samples tend to have larger fractions of BALQSOs (Dai et al. 2008). There has been some debate over whether selection biases in the optical make one underestimate the fraction of BALQSOs (Krolik \& Voit 1998). Essentially none of the BALs discovered with the F2MS survey would have been discovered with the SDSS color selection techniques (except for serendipitous spectra or searches for high-redshift quasars). Hence, our deduced fraction of BALs is too high (63\%) and even the conservative fraction of $40 \%$ deduced by Dai et al. (2008) and Shankar et al. (2008) is quite large. It becomes difficult to explain these nature objects as a pure orientation effect as the wind opening angle would be very large. However, since we are biased in only looking at dust-reddened objects, the dust could be present in the BAL clouds themselves, and we would naturally only find such a large number of BALs in objects that are reddened. Also, in the orientation picture, the highly collimated BAL winds could be grazing the dust torus, meaning 
that they are naturally redder than usual. These caveats aside, we believe that with such large dust reddenings, the evidence for mergers at lower redshift for similarly selected objects and the large intrinsic fraction lead us to believe that BALQSOs are in an early evolutionary phase in the lifetime of a quasar. Their powerful winds might be the quenchers of star formation in the host galaxy, often invoked as quasar feedback (e.g., Silk \& Rees 1998; Kauffmann \& Haehnelt 2000; Li et al. 2007).

The "youth hypothesis" for LoBALs fits very well with the picture of dust-reddened quasars being an evolutionary step in the lifetime of a quasar. The results from the hydrodynamic simulations predict that there is some amount of quasar feedback seen as a kind of "blowout" just after the phase where the merger has coalesced and the quasar is starting to ignite (albeit, enshrouded in dust; Hopkins et al. 2008). Whether this quasar feedback is actually done by a quasar wind that produces BALtype spectra is not clear yet though there is some evidence that LoBAL winds can extend into the host galaxy (de Kool et al. 2002). Recent poststarburst galaxies also show evidence for massive outflows which must have originated in a quasar when the starburst was still young (Tremonti et al. 2007).

The authors wish to thank Bryn Feldman for help with the first selections of this catalog and for help in carrying out the observations at Lick $3 \mathrm{~m}$ telescope. We are also thankful for the helpful comments from an anonymous referee on this paper. This work was partly performed under the auspices of the US Department of Energy by the Lawrence Livermore National Laboratory under contract no. DE-AC52-07NA27344.

This publication makes use of data products from the 2MASS, which is a joint project of the University of Massachusetts and the Infrared Processing and Analysis Center/California Institute of Technology, funded by the National Aeronautics and Space Administration and the National Science Foundation

Funding for the SDSS has been provided by the Alfred P. Sloan Foundation, the Participating Institutions, the National Aeronautics and Space Administration, the National Science Foundation, the US Department of Energy, the Japanese Monbukagakusho, and the Max Planck Society.

\section{REFERENCES}

Alonso-Herrero, A., et al. 2006, ApJ, 640, 167

Altschuler, D. R. 1986, A\&AS, 65, 267

Amirkhanyuan, V. R., Gorshkov, A. G., \& Ipatov, A. V. 1988, SoSAO, 58, 41

Antonucci, R. 1993, ARA\&A, 31, 473

Ballantyne, D. R., Shi, Y., Rieke, G. H., Donley, J. L., Papovich, C., \& Rigby, J. R. 2006, ApJ, 653, 1070

Becker, R. H., Gregg, M.D, Hook, I. M., McMahon, R. G., White, R. L., \& Helfand, D. J. 1997, ApJ, 479, 93

Becker, R. H., White, R. L., Gregg, M. D., Brotherton, M. S., \& LaurentMuehleisen, S. A. 2000, ApJ, 538, 72

Becker, R. H., White, R. L., \& Helfand, D. J. 1995, ApJ, 450, 559

Becker, R. H., et al. 2001, ApJS, 135, 227

Beers, T. C., Wilhelm, R., Doinidis, S. P., \& Mattson, C. J. 1996, ApJS, 103, 433

Boroson, T. A., \& Meyers, K. A. 1992, ApJ, 397, 442

Bregman, J. N., et al. 1984, ApJ, 276, 454

Brinkmann, W., Siebert, J., Reich, W., Fuerst, E., Reich, P., Voges, W., Truemper, J., \& Wielebinski, R. 1995, A\&AS, 109, 147

Brotherton, M. S., Tran, H. D., Becker, R. H., Gregg, M. D., LaurentMuehleisen, S. A., \& White, R. L. 2001, ApJ, 546, 775

Casebeer, D., Baron, E., Leighly, K., Jevremovic, D., \& Branch, D. 2008, ApJ, 676,857

Cutri, R. M., Nelson, B. O., Kirkpatrick, J. D., Huchra, J. D., \& Smith, P. J. 2001, in ASP Conf. Ser. 232, The New Era of Wide Field Astronomy, ed. R. G. Clowes, A. J. Adamson, \& G. E. Bromage (San Francisco, CA: ASP), 78
Czerny, B., Li, J., Loska, Z., \& Szczerba, R. 2004, MNRAS, 348, 54

Dai, X., Shankar, F., \& Sivakoff, G. R. 2008, ApJ, 672, 108

Dallacasa, D., Stanghellini, C., Centonza, M., \& Fanti, R. 2000, A\&A, 363, 887

de Grijp, M. H. K., Keel, W. C., Miley, G. K., Goudfrooij, P., \& Lub, J. 1992, A\&AS, 96, 389

de Kool, M., Becker, R. H., Arav, N., Gregg, M. D., \& White, R. L. 2002, ApJ, 570,514

Di Matteo, T., Srpingel, V., \& Hernquist, L. 2005, Nature, 433, 604

Farrah, D., Lacy, M., Priddey, R., Borys, C., \& Afonso, J. 2007, ApJ, 662, L59

Fitzpatrick, E. L. 1999, PASP, 111, 63

Gallagher, S. C., Brandt, W. N., Chartas, G., Priddey, R., Garmire, G. P., \& Sambruna, R. M. 2006, ApJ, 544, 709

Gallagher, S. C., Hines, D. C., Blaylock, M., Priddey, R. S., Brandt, W. N., \& Egami, E. E. 2007, ApJ, 665, 157

Gaskell, C. M., Goosmann, R. W., Antonucci, R. R. J., \& Whysong, D. J. 2004, ApJ, 616, 147

Gilli, R., Comastri, A., \& Hasinger, G. 2007, A\&A, 463, 79

Glikman, E., Gregg, M. D., Lacy, M., Helfand, D. J., Becker, R. H., \& White, R. L. 2004, ApJ, 607, 60

Glikman, E., Helfand, D. J., White, R. L., Becker, R. H., Gregg, M. D., \& Lacy, M. 2007, ApJ, 667, 673

Gregg, M. D., Lacy, M., White, R. L., Glikman, E., Helfand, D., Becker, R. H., \& Brotherton, M. S. 2002, ApJ, 564, 133

Hall, P. B., et al. 2002, ApJS, 141, 267

Hao, L., Weedman, D. W., Spoon, H. W. W., Marshall, J. A., Levenson, N. A., Elitzur, M., \& Houck, J. R. 2007, ApJ, 655, L77

Hao, L., et al. 2005, AJ, 129, 1795

Harris, D. E., Kapahi, V. K., \& Ekers, R. D. 1980, A\&AS, 39, 215

Heckman, T. M., Armus, L., \& Miley, G. K. 1987, AJ, 93, 276

Henstock, D. R., Browne, I. W. A., Wilkinson, P. N., Taylor, G. B., Vermeulen, R. C., Pearson, T. J., \& Readhead, A. C. S. 1995, ApJS, 100, 1

Hickox, R., et al. 2007, ApJ, 671, 1365

Hook, I. M., McMahon, R. G., Irwin, M. J., \& Hazard, C. 1996, MNRAS, 282, 1274

Hopkins, P. F., Hernquist, L., Cox, T. J., \& Keres, D. 2008, ApJ, 175, 356

Hopkins, P. F., Hernquist, L., Cox, T. J., Robertson, B., Di Matteo, T., \& Springel, V. 2006, ApJ, 638, 700

Hopkins, P. F., Hernquist, L., Martini, P., Cox, T. J., Robertson, B., Di Matteo, T., \& Springel, V. 2005, ApJ, 625, 71

Hutchings, J. B., Cherniawsky, A., Cutri, R. M., \& Nelson, B. O. 2006, AJ, 131 , 680

Hutchings, J. B., Maddox, N., Cutri, R. M., \& Nelson, B. O. 2003, AJ, 126, 63

Kauffmann, G., \& Haehnelt, M. 2000, MNRAS, 311, 576

Khrutskaya, E. V., Khovritchev, M. Yu., \& Bronnikova, N. M. 2004, A\&A, 418, 357

Kim, D.-C., \& Sanders, D. B. 1998, ApJS, 119, 41

Kirkpatrick, J. D., et al. 1999, ApJ, 519, 802

Knigge, C., Scaringi, S., Goad, M. R., \& Cottis, C. E. 2008, MNRAS, 386, 1426

Krolik, J. H., \& Voit, G. M. 1998, ApJ, 497, L5

Lacy, M., Gregg, M., Becker, R. H., White, R. L., Glikman, E., Helfand, D., \& Winn, J. N. 2002, AJ, 12, 2925

Lacy, M., Petric, A. O., Sajina, A., Canalizo, G., Storrie-Lombardi, L. J., Armus, L., Fadda, D., \& Marleau, F. R. 2007, AJ, 133, 186

Lacy, M., et al. 2004, ApJS, 154, 166

Lehár, J., Buchalter, A., McMahon, R. G., Kochanek, C. S., \& Muxlow, T. W. B. 2001, ApJ, 547, 60

Li, Y., et al. 2007, ApJ, 665, 187

Liske, J., Lemon, D. J., Driver, S. P., Cross, N. J. G., \& Couch, W. J. 2003, MNRAS, 344, 307

Low, F. J., Cutri, R. M., Huchra, J. P., \& Kleinmann, S. G. 1988, ApJ, 327, 41

Machalski, J. 1978, Acta Astron., 28, 367

Machalski, J. 1998, A\&AS, 128, 153

Machalsky, J., \& Maslowski, J. 1982, AJ, 87, 1132

Marble, A. R., Hines, D. C., Schmidt, G. D., Smith, P. S., Surace, J. A., Armus, L., Cutri, R. M., \& Nelson, B. O. 2003, ApJ, 590, 707

Martínez-Sansigre, A., Rawlings, S., Lacy, M., Fadda, D., Jarvis, M. J., Marleau, F. R., Simpsin, C., \& Willot, C. J. 2006, MNRAS, 370, 1479

Martínez-Sansigre, A., Rawlings, S., Lacy, M., Fadda, D., Marleau, F. R., Simpson, C., Willott, C. J., \& Jarvis, M. J. 2005, Nature, 436, 666

Monaco, L., Bellazzini, M., Bonifacio, P., Buzzoni, A., Ferraro, F. R., Marconi, G., Sbordone, L., \& Zaggia, S. 2007, A\&A, 464, 201

Odewahn, S. C., \& Aldering, G. 1995, AJ, 110, 2009

Petrosian, V. 1976, ApJ, 209, L1

Polletta, M., Weedman, D., Hoenig, S., Lonsdale, C. J., Smith, H. E., \& Houck, J. 2008, ApJ, 675, 960

Polletta, M., et al. 2007, ApJ, 663, 81

Reichard, T. A., et al. 2003a, AJ, 126, 2594 
Richards, G. T., et al. 2003b, AJ, 126, 1131

Richards, G. T., et al. 2004, ApJS, 155, 257

Richards, G. T., et al. 2006, AJ, 131, 2766

Richards, G. T., et al. 2009, AJ, 137, 3884

Rigby, J. R., Rieke, G. H., Donley, J. L., Alonso-Herrero, A., \& Pérez-González, P. G. 2006, ApJ, 645, 115

Sanders, D. B., Soifer, B. T., Elias, J. H., Madore, B. F., Matthews, K., Neugebauer, G., \& Scoville, N. Z. 1988, ApJ, 325, 74

Satyapal, S., Vega, D., Dudik, R. P., Abel, N. P., \& Heckman, T. 2008, ApJ, 677, 926

Schmidt, M. 1963, Nature, 197, 1040

Schmidt, G. D., Smith, P. S., Hines, D. C., Tremonti, C. A., \& Low, F. J. 2007, ApJ, 666, 784

Schneider, D. P., et al. 2003, AJ, 126, 2579

Schneider, D. P., et al. 2005, AJ, 130, 367

Shankar, F., Dai, X., \& Sivakoff, G. 2008, ApJ, 687, 859

Shi, Y., et al. 2006, ApJ, 653, 127

Shi, Y., et al. 2007, ApJ, 669, 841

Silk, J., \& Rees, M. J. 1998, A\&A, 331, 1

Smith, P. S., Schmidt, G. D., Hines, D. C., Cutri, R. M., \& Nelson, B. O. 2002, ApJ, 569, 23

Snellen, I. A. G., McMahon, R. G., Dennett-Thorpe, J., Jackson, N., Mack, K.-H., \& Xanthopoulos, E. 2001, MNRAS, 325, 1167

Snellen, I. A. G., McMahon, R. G., Hook, I. M., \& Browne, I. W. A. 2002, MNRAS, 329, 700

Skrutskie, M. F., et al. 1995, A\&AS, 187, 7507
Sprayberry, D., \& Foltz, C. B. 1992, ApJ, 390, 39

Stern, D., et al. 2007, ApJ, 663, 677

Szokoly, G. P., et al. 2004, ApJS, 155, 271

Tabara, H., Kato, T., Inoue, M., \& Aizu, K. 1984, PASJ, 36, 297

Treister, E., \& Urry, C. M. 2006, ApJ, 652, 79

Treister, E., et al. 2004, ApJ, 616, 123

Tremonti, C. A., Moustakas, J., \& Diamond-Stanic, A. M. 2007, ApJ, 663, 77

Trump, J., et al. 2006, ApJS, 165, 1

Urrutia, T., Lacy, M., \& Becker, R. H. 2008, ApJ, 674, 80

Urrutia, T., Lacy, M., Gregg, M. D., \& Becker, R. H. 2005, ApJ, 627, 75

Urry, C. M., \& Padovani, P. 1995, PASP, 107, 803

Véron-Cetty, M.-P., \& Véron, P. 2006, A\&A, 455, 773

Voit, G. M., Weynmann, R. J., \& Korista, K. T. 1993, ApJ, 413, 95

Weymann, R. J., Morris, S. L., Foltz, C. B., \& Hewett, P. C. 1991, ApJ, 373, 23

White, R. L., Helfand, D. J., Becker, R. H., Glikman, E., \& de Vries, W. 2007, ApJ, 654, 99

White, R. L., Helfand, D. J., Becker, R. H., Gregg, M. D., Postman, M., Lauer, T. R., \& Oegerle, W. 2003, AJ, 126, 706

Wilkinson, P. N., Browne, I. W. A., Patnaik, A. R., Wrobel, J. M., \& Sorathia, B. 1998 , MNRAS, 300, 790

Wills, B. J., et al. 1983, ApJ, 274, 62

Windhorst, R. A., et al. 1991, ApJ, 380, 362

Winn, J. N., Lovell, J. E. J., Chen, H.-W., Fletcher, A. B., Hewitt, J. N., Patnaik, A. R., \& Schechter, P. L. 2002, ApJ, 564, 143

Zakamska, N. L., et al. 2003, AJ, 126, 2125

Zakamska, N. L., et al. 2006, AJ, 132, 1496 